\title{
Estado de la investigación educativa en torno a la germinación de semillas: entre prácticas tradicionales y la perspectiva de la restauración ecológica
}

\section{State of educational research on seed germination: between traditional practices and the ecologial resotration perspective}

\section{Estado da pesquisa em germinação de sementes: entre as práticas tradicionais e a perspectiva da restauração ecológica}

\author{
María Josefa Rassetto (mjrassetto@ hotmail.com) \\ Universidad Nacional del Comahue \\ Julieta Farina (julietafarina@yahoo.com.ar) \\ Universidad Nacional del Comahue \\ Daniel Peréz (danielrneuquen@gmail.com) \\ Universidad Nacional del Comahue
}

Resumen: Este trabajo presenta el tramo inicial de una tesis doctoral ${ }^{1}$ que incluye el estudio de los antecedentes en investigación educativa sobre la germinación de semillas. Se expone una revisión de artículos publicados en revistas indizadas, considerando investigaciones realizadas en distintos niveles educativos. Se encontraron 25 artículos, que se estudiaron según las dimensiones analíticas: editorial, enseñanza de la germinación, procedencia biogeográfica de la planta, dormición de la semilla y tema del trabajo. Los resultados muestran que su enseñanza se basa principalmente en el uso de germinadores de semillas de Phaseolus vulgaris (porotos o frijoles), en la aplicación agrícola de semillas y en su mayoría no mencionan mecanismos de dormición o la forma de superarlos. Se concluye que el interés en los artículos analizados se centra en el proceso de emergencia de la plántula, dejando fuera las diferencias que se presentan en distintas especies, el origen biogeográfico, la relación de la germinación con las adaptaciones, los conocimientos socioculturales, o la posible aplicación a la restauración ecológica.

Palabras claves: alfabetización científica; germinación de semillas; enfoque relacional; restauración ecológica.

Abstract: This work presents the initial section of a doctoral thesis that includes the study of the background in educational research on seed germination. It exposed a

${ }^{1}$ Tesis Doctoral inscripta en el Doctorado en Enseñanza de las Ciencias Exactas y Naturales de la Facultad de Ingeniería, Universidad Nacional del Comahue, Argentina. Tesista Julieta Farina; Directora Dra. María Josefa Rassetto; Co-Director Dr. Daniel Roberto Pérez.

Recebido em: 30/05/2021

Aceite em: 13/08/2021 
bibliographic review of articles published in indexed journals, including studies carried out at different educational levels. Twenty-five articles were found, that were studied according to the analytical dimensions: editorial, relationship with the teaching of germination, biographic origin of plant, mention of seed dormancy and the central topic of the work. The results show that the teaching of the germination process is mainly based on the use of containers or glass containers for seed germination and seeds of Phaseolus vulgaris (known as beans), or on the agricultural application of seeds and that most of them do not mention dormancy alleviation treatments. It is concluded that the interest in the research focuses on the seedling emergence process, without considering differences that occur in different native's species, the biogeographical origin, or the relationship of germination with the plant adaptations, sociocultural knowledge, or its application to ecological restoration.

Keywords: scientific literacy; seed germination; relational approach; ecological restoration.

Resumo: Este trabalho apresenta a seção inicial de uma tese de doutorado que inclui o estudo dos antecedentes da pesquisa educacional em germinação de sementes. Expôs uma revisão de artigos publicados em revistas indexadas, considerando pesquisas realizados em diferentes níveis de ensino. Foram encontrados 25 artigos, que foram estudados de acordo com as dimensões analíticas: editorial, relação com o ensino de germinação, origem biogeográfica da planta, menção à dormência da semente e tema central do trabalho. Os resultados mostram que o ensino de germinação é baseado principalmente na utilização de germinadores de sementes de Phaseolus vulgaris (conhecidos como feijões), ou na aplicação agrícola de sementes e que a maioria delas não menciona mecanismos de dormência ou ruptura da dormência. Conclui-se que o interesse na pesquisa centra-se no processo de emergência de plântulas, sem considerar as diferenças que ocorrem nas diferentes espécies nativas, a origem biogeográfico, ou a relação da germinação com as adaptações das plantas, conhecimentos socioculturais, ou sua aplicação à restauração ecológica.

Palavras-chave: alfabetização científica; germinação de sementes; abordagem relacional; restauração ecológica.

\section{INTRODUCCIÓN}

La germinación es un contenido presente en los diseños curriculares de varios países del mundo, tanto para el nivel infantil (HILLINSON, 1998; REAL DECRETO 1603/2006; DISEÑO CURRICULAR PARA LA EDUCACIÓN INICIAL, 2019; RASSETTO; FARINA; PÉREZ, 2021²), como para el primario (LINK-PÉREZ, 2013;

\footnotetext{
${ }^{2}$ RASSETTO, M., FARINA, M.J., PÉREZ, D.R. La restauración ecológica como contenido escolar en
} los diseños curriculares de las Provincias de Neuquén y Río Negro. Argentina. MULTEQUINA (Latin

Recebido em: 30/05/2021

Aceite em: $13 / 08 / 2021$ 
DISEÑO CURRICULAR NIVEL PRIMARIO, 2011; REAL DECRETO 126/2014).

Este tópico tiene relevancia tanto por los modelos científicos que implica dentro del campo de la Biología (VIDAL; MEMBIELA, 2015), las potencialidades para desarrollar habilidades procedimentales o procesos científicos (HAMMANN; HOL PHAN; EHMER; GRIMM, 2008), y para favorecer actitudes para la recuperación de los ambientes degradados (PÉREZ et al., 2019).

Dada la relevancia del tema, es de esperar que su enseñanza esté acompañada de numerosas investigaciones educativas que permitan interpretar la germinación desde variadas dimensiones y que tengan en cuenta aspectos epistemológicos, metodológicos, conceptuales, y ambientales. Con base en esta premisa, en este trabajo se efectúa la revisión de publicaciones sobre el tema de la germinación, considerando que el análisis de abordajes ya efectuados sobre un tema desde la perspectiva del proceso de investigación educativa en el campo de la Didáctica de las Ciencias Naturales permite, no sólo sistematizar lo que se sabe de un determinado problema de estudio, sino también detectar los vacíos de conocimiento (ACHILLI, 2000). Asimismo "la revisión crítica de los antecedentes de investigación es una forma de seguir avanzando en el campo de estudio y repercutir de forma creciente y positiva en las prácticas educativas" (CACHAPUZ et al., 2006, p.171). En el marco de una tesis de doctorado, es fundamental hacer una exhaustiva revisión y análisis de los antecedentes en investigación, tanto para dar cuenta de lo que se ha avanzado sobre un determinado tema de estudio, como para detectar posibles vacíos y proponer avances en la enseñanza significativa y relacional de las Ciencias Naturales. La presente revisión de antecedentes se encuadra en el marco de un programa mayor de investigación que tiene como finalidad construir, de manera colaborativa con docentes de educación infantil (preescolar), propuestas didácticas que promuevan la alfabetización científica en torno a las adaptaciones de las plantas y la germinación.

En este contexto, este trabajo tuvo como objetivos particulares, a) ofrecer una revisión de las diversas investigaciones llevadas a cabo sobre el contenido "germinación" en diversos niveles educativos, como parte de la etapa inicial de la tesis

American Journal on Natural Resources). Número especial sobre Restauración ecológica en ecosistemas aridos, semi-aridos y subhumedos (en prensa). 2021.

Recebido em: 30/05/2021

Aceite em: 13/08/2021 
doctoral, b) analizar los resultados que se mencionan, c) discutir tanto los aportes que se realizan desde ámbitos académicos a la enseñanza de este tema, como las posibilidades de abordaje que podrían ser investigadas en el futuro.

\section{MARCO TEÓRICO}

\section{La germinación, un contenido clave de la Biología y la Ecología}

Desde el punto de vista biológico y ecológico, las semillas se consideran la unidad de reproducción sexual de las plantas fanerógamas (BASKIN; BASKIN, 2014) y son clave para comprender tanto la dinámica de las poblaciones como la fase inicial de la colonización en el proceso de sucesión primaria y secundaria (DUNCAN et al., 2019).

La germinación de la semilla ocurre cuando emerge la radícula y generalmente se da con el ingreso de agua, que activa el metabolismo del embrión (BASKIN; BASKIN; LI, 2000). La mayoría de las especies vegetales presentan mecanismos de dormición. Una semilla con dormición es aquella que no tiene la capacidad de germinar bajo ninguna combinación de factores ambientales normales y solo puede hacerlo cuando es removida la causa que limita o impide la activación de su metabolismo (BASKIN; BASKIN, 2004). Esta característica de las semillas es más frecuente en las zonas áridas y semiáridas, en donde aproximadamente el 85-90\% de las especies tienen dormancia que bloquea la germinación bajo condiciones desfavorables y las permite cuando las condiciones ambientales brindan mayores chances de sobrevivir (RODRÍGUEZ ARAUJO; PÉREZ; ARONSON; CROSS, 2021³), “afinando" la germinación a las condiciones del ambiente (BASKIN, 2003; DUNCAN et al. 2019). Las semillas con dormancia no germinan hasta que naturalmente se rompe el mecanismo que la limita por acción ambiental o se promueve el proceso mediante técnicas pre-germinativas, algunas de las cuales son la escarificación (por ejemplo, raspado, tratamientos con ácidos) y la estratificación (por ejemplo, tratamiento frio húmedo o alternancias de temperatura) (RODRIGUEZ ARAUJO; PÉREZ; BONVISSUTO, 2017).

\footnotetext{
${ }^{3}$ RODRÍGUEZ ARAUJO, M.E.: PÉREZ, D.R.; ARONSON, J; CROSS, A.T. Filling gaps in seed germination and species selection: Work in progress for dryland restoration in Argentina. MULTEQUINA (Latin American Journal on Natural Resources). Número especial sobre Restauración ecológica en ecosistemas áridos, semiáridos y subhúmedos (en prensa). 2021.
}

Recebido em: 30/05/2021

Aceite em: 13/08/2021 
Teniendo en cuenta entonces que, la germinación de las semillas depende del ambiente del cual forman parte, y que en su mayoría necesitan superar un proceso de dormición, a la hora de enseñar sobre germinación se espera más que un abordaje meramente descriptivo a nivel morfológico o de la fisiología; las investigaciones sobre enseñanza en el tema debieran considerar tanto los aspectos bio-ecológicos como de diversidad biológica y a la germinación como una adaptación particular de las plantas a diferentes condiciones ambientales y regiones biogeográficas.

\section{Enfoques de enseñanza y germinación}

La enseñanza de la germinación, como contenido de las Ciencias Naturales, puede abordarse desde diferentes lógicas, perspectivas o enfoques. Desde las disciplinas de las Ciencias Biológicas es posible poner el acento en los datos y conceptos de los procesos morfofisiológicos que implican la germinación de la semilla (POZO; CRESPO, 2000) y los factores ambientales como humedad, temperatura, y otros, que la preceden (JEWELL, 2002).

Otro enfoque posible, parte del conocimiento relacional, que se promueve en el ámbito escolar para favorecer la interpretación del mundo, y la participación autónoma y crítica de los ciudadanos en la gestión de problemas ambientales (GARCÍA, 1994; GARCÍA; PORLÁN ARIZA, 2000; POZZOLI, 2007). Esta lógica relacional, constituye uno de los fundamentos de los diseños curriculares en la Argentina, Latinoamérica y otros lugares del mundo (KAUFMANN; SERULNICOFF, 2000; CRUZ GUZMÁN; PUIG; GARCÍA-CARMONA, 2020;) que promueven la indagación del ambiente en su complejidad y aportan a que los niños y niñas sean partícipes activos de la toma de decisiones sobre problemáticas ambientales. Desde esta perspectiva, el tema de la germinación puede articularse con la Educación Ambiental, entendida en forma relacional. Esta corriente, ha cobrado fuerza con rasgos particulares en América Latina y Caribe (TRELLEZ, 2006) con la incorporación de la educación popular y comunitaria recuperando planteamientos de Paulo Freire, las formulaciones de Enrique Leff (2004) en torno al diálogo de saberes y la racionalidad ambiental, y la preocupación por 
proteger su mega-biodiversidad desde vertientes de la Ecología (FREIRE, 1969; CALIXTO FLORES, 2010; PEREZ; RASSETO; FARINA, 20214).

Por otra parte, la alfabetización científica en la educación básica, se centra en favorecer la indagación del ambiente, iniciar a los niños y las niñas en la identificación de problemas ambientales y promover el reconocimiento de la diversidad biológica y de relaciones entre seres vivos y con su ambiente (NÚCLEOS DE APRENDIZAJE PRIORITARIOS, 2004; LINK PÉREZ; SCHUSSLER, 2013; ALVES, 2016; CRUZ GUZMÁN; PUIG; GARCÍA CARMONA, 2020). Esta perspectiva es acorde con la lógica relacional de la Didáctica de las Ciencias Naturales.

En el marco del enfoque relacional, la educación ambiental y la indagación del ambiente, se han desarrollado experiencias educativas innovadoras tanto en el ámbito de la educación formal como no formal, y a nivel regional como mundial. Algunas de estas experiencias se centraron en lo que Lepold (1949), denominó ética de la tierra, que se basa en transformar la relación entre las personas y la naturaleza. Entre ellas encontramos las desarrolladas en Wisconsin con 150 escuelas incluidas en el programa earth partnership (MCCANN; MILLER, 2004); las propuestas educativas para campesinos que habitan la selva Amazónica (GARZÓN; RODRÍGUEZ LEÓN; CECCON; PÉREZ, 2020); el trabajo con residentes urbanos de zonas áridas de la norpatagonia Argentina (PÉREZ et al., 2019); la inclusión de minorías étnicas y migrantes en Canadá (NEWMAN, 2011) y la "rehabilitación humana" de prisioneros de cárceles de Río de Janeiro, Brasil (MARQUES DE ABREU et al., 2017), entre otras.

\section{METODOLOGÍA}

Con el fin de ubicar las publicaciones en investigación educativa a nivel mundial, que traten sobre la enseñanza del proceso de germinación de semillas en ámbitos educativos, se recurrió a la consulta de los principales buscadores de publicaciones de investigación en educación de acceso libre, siguiendo a Romera (2014), quien propone

\footnotetext{
${ }^{4}$ Pérez, Rassetto, Farina. Relationships between ecological restoration and environmental education. A critical view from Enrique Leff's conceptual framework. Desenvolvimento e meio ambiente. Em prensa. 2021.
} 
el análisis según procedimientos de metodología documental. Se buscó bibliografía en las plataformas y/o redes web: SciELO, ResearchGate, Academia.edu y Google Scholar. Los términos de la cadena de búsqueda se escribieron tanto en idioma inglés, castellano y portugués. Los mismos fueron: teaching seed germination, teaching seed germination, education about seed germination, sus equivalentes en castellano: enseñanza de la germinación de semillas, enseñanza de la germinación y educación en germinación de semillas y en portugués: ensino germinação sementes, ensino germinação y educação germinação. Como surge de los términos utilizados en la búsqueda, el trabajo se inició haciendo foco en la enseñanza específica de la germinación, pero la escasa presencia de publicaciones con esta especificidad, hizo que se seleccionen trabajos que, si bien no tratan en forma exclusiva la enseñanza de la germinación, incluyeron el tema en el marco de otros tópicos de la enseñanza de las Ciencias Naturales. Una vez realizada la búsqueda, del nivel universitario y terciario sólo se seleccionaron los trabajos referidos a la formación de profesores para la escuela inicial (preescolar), primaria y secundaria dado que son los futuros profesores que llevarán adelante sus prácticas en el sistema educativo obligatorio.

Los artículos fueron leídos y sistematizados en función de los tópicos: autores y/o autoras, año, revista, país de residencia de autores/as, y nivel educativo donde se desarrolló la investigación. Luego de la lectura exhaustiva, se construyeron dimensiones analíticas con categorías que permitieron un estudio en profundidad de cada artículo (STATU; BONIOLO; DALLE; ELBER, 2005). A continuación, se detalla cada dimensión con las categorías correspondientes:

Dimensión Editorial: refiere a qué tipo de trabajo se publica, atendiendo si se trata de una experiencia de enseñanza o de una investigación didáctica. Las categorías establecidas para esta dimensión fueron:

a. Experiencia didáctica: cuando se relata la puesta en marcha de una secuencia de actividades en un grupo de clase concreto. Se consideran dentro de esta categoría artículos que incorporan algún tipo de análisis luego de llevadas a cabo actividades en el aula.

Dentro de esta categoría, se diferenciaron además aquellos trabajos que fundamentaron la experiencia desde perspectivas y corrientes centradas en la 
enseñanza, de los que lo hacen desde perspectivas del aprendizaje. Se entiende a una corriente dentro de la didáctica como tendencias de ideas dentro del campo, que definen determinados modos de investigación y/o enseñanza, incluyendo la dimensión metodológica de ambos procesos.

b. Investigación educativa: son trabajos en los que se analizó un tópico particular sin hacer referencia a un grupo de clase concreto. A su vez, en esta categoría se diferenció entre publicaciones con énfasis en métodos de investigación cualitativos de las que tuvieron enfoque cuantitativo. Cabe señalar que, en la práctica de la investigación en Didáctica de las Ciencias Naturales, generalmente se realiza una triangulación que combina estrategias de recolección e interpretación de datos tanto cuali como cuantitativos (BRAVIN; PIEVI, 2008).

Relación con la enseñanza de la germinación de semillas: refiere a si el foco del trabajo estuvo puesto o no en la enseñanza de la germinación de semillas. De esta manera se establecieron como categorías:

a. Relación directa: para aquellos artículos en los que la enseñanza estuvo centrada en el tópico "germinación".

b. Relación indirecta: artículos en los que la enseñanza estuvo centrada en otros tópicos, pero incluyeron "germinación".

Procedencia biogeográfica de la planta: esta dimensión incluyó al hábitat de la planta en relación con la naturaleza y/o la sociedad, es decir, se consideró si eran plantas propias del ambiente natural (silvestres, nativas) o si son eran sembradas y/o cultivadas (FARINA; LOZADA; LADIO, 2008). Esta dimensión pudo ser analizada en aquellos artículos que mencionaron el nombre (común o científico) de la especie de planta sobre la que trabajó. De acuerdo al criterio de los autores de este trabajo, se consideraron como categorías:

a. Plantas silvestres, nativas: crecen en vida libre, sin que sean sembradas o cultivadas por humanos, y son originarias de la ecorregión o territorio biogeográfico.

b. Plantas cultivadas: son cultivadas en huertas o invernaderos y dependen de la acción del ser humano para que germinen y crezcan en esas condiciones. 
Mención respecto a mecanismos de dormición de las semillas: En esta dimensión se diferenciaron aquellos trabajos que señalan o mencionaron mecanismos de dormición de las semillas y los procesos para romper la misma. Las categorías fueron dicotómicas y señalaron presencia o ausencia.

Tema del trabajo: esta dimensión refiere a cuál es el tema central del trabajo, o sea, el contenido que consideró importante enseñar y/o aprender. Las categorías utilizadas para el análisis fueron:

a. Anatomía y fisiología de la germinación de semillas: incluye a los trabajos que ponen el centro de interés en la enseñanza y el aprendizaje en los conceptos anatómicos descriptivos y procesos fisiológicos que dan lugar a la germinación.

b. Habilidades procedimentales: ponen el centro de interés en la enseñanza y/o aprendizaje de los procesos de ciencia escolar como el planteamiento de hipótesis, la resolución de problemas, la indagación, la observación, el diseño experimental, el análisis de resultados y la elaboración de conclusiones (PORLÁN ARIZA, 1999; CRUJEIRAS PÉREZ; JIMÉNEZ ALEIXANDRE, 2015)

c. Ecología: se incluyeron dentro de esta categoría los trabajos que están centrados en la enseñanza y/o aprendizaje de conceptos de Ecología como, por ejemplo: dinámica de poblaciones/ o comunidades, conservación de ecosistemas, restauración ecológica, entre otros.

d. Concepciones profesorales/concepciones del estudiantado: se consideró a las concepciones como distintas teorías que las y los sujetos elaboran respecto de determinados conceptos (SUAREZ SILVA; FORTES BRAIBANTE, 2018; FARINA; ACUÑA; PÉREZ; RASSETTO, 2019). Dentro de esta categoría se incluyeron a los trabajos que abordan temáticas referidas a las concepciones de las y los docentes sobre la enseñanza de contenidos botánicos o del estudiantado sobre germinación.

e. Salud: esta categoría abarca los trabajos cuyo centro de interés está dado por la enseñanza de hábitos alimenticios para la salud.

f. Alfabetización agrícola: incluye a trabajos que refieren a temáticas sobre la enseñanza de contenidos agrícolas, tanto de cultivo de plantas como de crianza de animales. 


\section{RESULTADOS Y DISCUSIÓN}

El resultado de la búsqueda permitió analizar un total de veinticinco artículos, cuya sistematización se presenta en la tabla 1. Cabe aclarar que para los autores de los artículos numerados como 4, 17, 20 y 21, se encontraron dos artículos del mismo tema/contenido, por lo que se decidió analizar el publicado más recientemente.

Tabla 1. Sistematización de los artículos analizados, según autor, año de publicación, revista, país y nivel educativo, ordenadas según año de publicación creciente.

\begin{tabular}{|c|c|c|c|c|c|}
\hline $\mathrm{N}^{\circ}$ & Autores & Año & Revista & País & $\begin{array}{c}\text { Nivel } \\
\text { educativo }\end{array}$ \\
\hline 1 & F. Pascoe & 1994 & $\begin{array}{c}\text { The American Biology } \\
\text { Teacher }\end{array}$ & EEUU & Secundario \\
\hline 2 & R. Mabie y M. Baker & 1996 & $\begin{array}{c}\text { Journal of Agricultural } \\
\text { Education }\end{array}$ & EEUU & Primario \\
\hline 3 & J. Hillison & 1998 & $\begin{array}{c}\text { Journal of Agricultural } \\
\text { Education }\end{array}$ & EEUU & $\begin{array}{l}\text { Infantil y } \\
\text { Primario }\end{array}$ \\
\hline 4 & $\begin{array}{l}\text { M. Duarte y M. } \\
\text { Gonçalves }\end{array}$ & 1999 & Boletín das Ciencias & Portugal & Primario \\
\hline 5 & N. Jewell & 2002 & $\begin{array}{c}\text { Journal of Biological } \\
\text { Education }\end{array}$ & Inglaterra & $\begin{array}{l}\text { Infantil y } \\
\text { Primário }\end{array}$ \\
\hline$\theta_{6}$ & Stosheau-Wen Lin & 2004 & $\begin{array}{c}\text { International Journal of } \\
\text { Science and Mathematics } \\
\text { Education }\end{array}$ & Taiwan & Secundario \\
\hline 7 & $\begin{array}{c}\text { B. Jeanpierre, K. } \\
\text { Oberhauser y C. Freeman }\end{array}$ & 2005 & $\begin{array}{l}\text { Journal of Research in } \\
\text { Science Teaching }\end{array}$ & EEUU & Secundario \\
\hline 8 & $\begin{array}{c}\text { A. Zohar y S. Aharon- } \\
\text { Kravetsky }\end{array}$ & 2005 & $\begin{array}{l}\text { Journal of Research in } \\
\text { Science Teaching }\end{array}$ & Israel & Secundario \\
\hline 9 & $\begin{array}{l}\text { R. Fernández Manzanal y } \\
\text { L. M. Rodríguez Barreiro }\end{array}$ & 2006 & Alambique & España & Infantil \\
\hline 10 & $\begin{array}{l}\text { M. Hammann, T. T. Hol } \\
\text { Phan, M. Ehmer y T. } \\
\text { Grimm }\end{array}$ & 2008 & $\begin{array}{c}\text { Journal of Biological } \\
\text { Education }\end{array}$ & Alemania & Secundario \\
\hline 11 & A. Zohar y A. B. David & 2008 & Metacognition Learning & Israel & Secundario \\
\hline 12 & D. M. Pecaski McLennan & 2010 & Early Childhood Education & Canadá & Infantil \\
\hline 13 & E. J. Ju y J. G. Kim & 2011 & $\begin{array}{c}\text { Journal of Biological } \\
\text { Education }\end{array}$ & Korea & Primario \\
\hline
\end{tabular}




\begin{tabular}{|c|c|c|c|c|c|}
\hline 14 & $\begin{array}{c}\text { M. Zhang, J. Parker, J. } \\
\text { Eberhardt y S. } \\
\text { Passalacqua }\end{array}$ & 2011 & $\begin{array}{c}\text { Journal Science Education } \\
\text { Technology }\end{array}$ & EEUU & Infantil \\
\hline 15 & $\begin{array}{l}\text { M. A. Link-Pérez y E. E. } \\
\text { Schussler. }\end{array}$ & 2013 & Plant Science Bulletin & EEUU & $\begin{array}{c}\text { Formación } \\
\text { docente }\end{array}$ \\
\hline 16 & $\begin{array}{l}\text { N. R. Stefanine, F. Silva } \\
\text { Darosci Brito, F. Castro y } \\
\text { J. Alves de Almeida }\end{array}$ & 2011 & Revista Querubim & Brasil & Secundario \\
\hline 17 & M. Vidal y P. Membiela & 2015 & $\begin{array}{c}\text { Journal of Biological } \\
\text { Education }\end{array}$ & España & $\begin{array}{l}\text { Formación } \\
\text { docente }\end{array}$ \\
\hline 18 & $\begin{array}{l}\text { A. da Silva Fontes, C. } \\
\text { Cargnin, Michel C. } \\
\text { Batista, F. Peres Ramos, } \\
\text { R. da Silva Gonzalez y P. } \\
\text { Altoé Fusinato }\end{array}$ & 2015 & Ensino, Saúde e Ambiente & Brasil & Secundario \\
\hline 19 & A. Alves & 2016 & $\begin{array}{c}\text { Editura Universitatii } \\
\text { "Alexandru Ioan Cuza" }\end{array}$ & Portugal & Infantil \\
\hline 20 & $\begin{array}{l}\text { M. T. Olvera Hernández, } \\
\text { Á.D. López y Mota y O. } \\
\text { E. Tamayo Alzate }\end{array}$ & 2016 & $\begin{array}{c}\text { Revista Tecné, Episteme y } \\
\text { Didaxis: TED. }\end{array}$ & México & $\begin{array}{l}\text { Formación } \\
\text { docente }\end{array}$ \\
\hline 21 & $\begin{array}{c}\text { F. J. Ruiz Ortega y D. A. } \\
\text { Zapata Mira }\end{array}$ & 2017 & $\begin{array}{l}\text { Bio - grafía. Escritos sobre } \\
\text { la Biología y su Enseñanza }\end{array}$ & Colombia & Primario \\
\hline 22 & $\begin{array}{l}\text { D. da Silva Alves, R. de } \\
\text { Souza Poletto y M. } \\
\text { Meneghello Passos }\end{array}$ & 2019 & Revista Ciências \& Ideas & Brasil & $\begin{array}{c}\text { Formación } \\
\text { docente }\end{array}$ \\
\hline 23 & $\begin{array}{l}\text { R. I. da Costa Güllich } \\
2\end{array}$ & 2020 & Revista Insignare Scientia & Brasil & $\begin{array}{c}\text { Formación } \\
\text { docente }\end{array}$ \\
\hline 24 & $\begin{array}{l}\text { C. Fossati Ferri, J. } \\
\text { Bortolini, Tatiane } \\
\text { Eitelven, L. Terezinha } \\
\text { Zanol Sauer y G. } \\
\text { Brambatti Guzzo }\end{array}$ & 2020 & Scientia Cum Industria & Brasil & Primario \\
\hline 25 & $\begin{array}{l}\text { M. Ceballos Aranda, B. } \\
\text { Pérez-Bueno, M. Reina y } \\
\text { J. E. Vilchez }\end{array}$ & 2021 & Investigación en la Escuela & España & Primario \\
\hline
\end{tabular}

Fuente: Elaboración propia

Del total de las publicaciones estudiadas, siete fueron investigaciones que se desarrollaron en América del Norte, siete en Europa, siete en Latinoamérica y cuatro en Asia. El rango de años de las publicaciones abarcó, desde 1994 hasta 2021 y los niveles educativos en los que se desarrollaron, incluyeron el nivel infantil (16\%), primario (28\%), secundario (28\%) y de formación docente (20\%). El 8\% de los trabajos, estuvieron enfocados en la enseñanza tanto para la educación infantil como para la 
escuela primaria. Estos resultados reflejan que, tal como se señaló en la introducción, la enseñanza de la germinación es un tópico frecuente y de interés tanto en la educación infantil como en la primaria, niveles para los cuales se encontraron un total de catorce trabajos. También fue un tópico presente en el nivel secundario, si bien, como se discutirá más adelante, estuvo asociado a otras temáticas biológicas, salvo para los trabajos desarrollados en Brasil. Respecto a los autores de los artículos analizados, se contabilizan un total de 62 , uno de ellos repetido en uno de los trabajos.

Los resultados encontrados respecto de las dimensiones y categorías con las que se analizaron los artículos se sintetizan en la Tabla 2. Se observa que, para la dimensión editorial, la mayoría de los trabajos analizados pertenecen a la categoría experiencias didácticas $(76,00 \%)$, es decir, propuestas llevadas a cabo en un contexto de aula, que fueron analizados en función de la corriente que fundamenta el trabajo. La mayoría de las publicaciones consideradas en esta dimensión, fundamentan la experiencia desde diferentes corrientes didácticas centradas en la enseñanza (79\%). Entre estas corrientes se destaca la enseñanza por indagación (40\% de las experiencias didácticas), seguida por la enseñanza basada en el uso de pruebas (20\%) y las centradas fuera del aula y por resolución de problemas, con un 13,3\% de trabajos fundamentados desde cada una de esas corrientes. Se identificó un trabajo (lo que representa el 6,7\%) para la corriente de enseñanza basada en proyectos. Las experiencias fundamentadas desde el aprendizaje correspondieron a un $21 \%$ y entre las corrientes identificadas se encuentran: la evolución de modelos explicativos, la provocación del conflicto cognitivo y la construcción de estrategias metacognitivas por parte del estudiantado.

Tabla 2. Porcentaje de trabajos según las categorías evaluadas en cada dimensión.

\begin{tabular}{|c|c|c|}
\hline Dimensión & Categoría & Porcentaje \\
\hline \multirow{2}{*}{ Editorial } & $\begin{array}{c}\text { Experiencia } \\
\text { Didáctica }\end{array}$ & $76 \%$ \\
\cline { 2 - 3 } & $\begin{array}{c}\text { Investigación } \\
\text { Educativa }\end{array}$ & $24 \%$ \\
\hline \multirow{2}{*}{$\begin{array}{c}\text { Relación con } \\
\text { germinación }\end{array}$} & Directa & $56 \%$ \\
\cline { 2 - 3 } & Indirecta & $44 \%$ \\
\hline Tipo de planta & Silvestre & $12 \%$ \\
\hline
\end{tabular}




\begin{tabular}{|c|c|c|}
\hline \multirow{4}{*}{$\begin{array}{c}\text { Dormición de } \\
\text { las semillas }\end{array}$} & Cultivada & $76 \%$ \\
\cline { 2 - 3 } & No menciona & $12 \%$ \\
\cline { 2 - 3 } & Presente & $20 \%$ \\
\hline \multirow{4}{*}{$\begin{array}{c}\text { Tema del } \\
\text { Trabajo }\end{array}$} & $\begin{array}{c}\text { Anatomía y } \\
\text { Fisiología }\end{array}$ & $80 \%$ \\
\cline { 2 - 3 } & $\begin{array}{c}\text { Habilidades } \\
\text { Procedimentales }\end{array}$ & $28 \%$ \\
\cline { 2 - 3 } & Ecología & $12 \%$ \\
\cline { 2 - 3 } & Concepciones & $12 \%$ \\
\cline { 2 - 3 } & Salud & $4 \%$ \\
\cline { 2 - 3 } & Agricultura & $8 \%$ \\
\hline
\end{tabular}

Fuente: Elaboración propia

Respecto a los artículos clasificados en la dimensión editorial como de investigación educativa se observa que, el $50 \%$ son de carácter cualitativo. Los trabajos de esta categoría refieren a: análisis curricular, técnicas de laboratorio utilizadas en ecología y concepciones docentes sobre enseñanza de tópicos botánicos. El $50 \%$ restante son de corte cuantitativo, con solo un trabajo que indaga las concepciones de estudiantes sobre procesos en las plantas y otro que evalúa las habilidades procedimentales, ambos en el nivel secundario. El trabajo restante, trata las concepciones de niños y niñas de escuela primaria e infantil sobre semillas y germinación.

Sobre la dimensión relación con la germinación, se reporta que, un poco más de la mitad de los trabajos tienen una relación con la germinación, directa $(56,00 \%)$ lo cual, sumado a la poca cantidad de artículos encontrados, señala una escasez en la producción en investigación centrada en la enseñanza de este tema. Los trabajos catalogados como de relación directa con la germinación, son en su mayoría experiencias de aula (86\%), tanto de nivel inicial como de primaria, y formación docente. Como se señaló más arriba para el nivel secundario, se encontró que solo dos trabajos $(10,5 \%)$ son experiencias de relación directa con la germinación y ambos están desarrollados en Brasil. Los trabajos categorizados como de relación indirecta, es decir no centrados en la enseñanza de la germinación, corresponden en su mayoría al nivel 
infantil y secundario (63\%) y están enfocados en otros tópicos como son el desarrollo de habilidades procedimentales y Ecología.

Al analizar la dimensión origen biogeográfico de la planta (figura 1), se observa que la enseñanza de la germinación está centrada en las plantas cultivadas (76,00\%). Dentro de estas plantas se destaca el uso de semillas de la especie Phaseolus vulgaris (conocidas como porotos, frijoles, judías) que se impone como el modelo de semilla para enseñar el tema, ya que se encontró en once de los trabajos analizados, lo que representa el $58 \%$ dentro de la categoría. Es interesante destacar que la estrategia de enseñanza que recurre frecuentemente a recipientes germinadores (normalmente frascos de vidrio o equivalentes) de Phaseolus vulgaris continúa siendo una referencia en artículos más actuales (por ejemplo, los de número de orden 15, 16, 17, 20, 21 y 24, de la tabla 1). Otras especies utilizadas que reportan los trabajos son: Lens culinaris (lentejas), Zea mays (maíz), Solanum lycopersicum (tomate), Curcubita maxima (calabaza), Cucumis melon (melón), Latuca sativa (lechuga), Malus domestica (manzana), entre otras; siempre con objetivos de alcanzar ideas generalizadoras sobre las condiciones de germinación (temperatura, humedad) y el proceso morfológico y/o fisiológico de emergencia de radícula, cotiledones y estructuras de la plántula.

Los trabajos que hacen referencia a plantas silvestres corresponden al 12,00\%, lo que podría evidenciar escasa relación de la germinación con la biodiversidad y el ambiente cercano en que habita el estudiantado. Asimismo, resulta llamativa la escasez de enfoques novedosos para la enseñanza del tema, como lo podrían ser la siembra de especies nativas y/o producción de las mismas para la restauración de ambientes degradados (MC CANN, 2011). Una excepción encontrada fue el artículo de Pascoe $\left(\mathrm{N}^{\circ} 1\right.$ en la tabla 1$)$, que aborda la enseñanza de la germinación de plantas silvestres, asociada a conceptos ecológicos y a una mirada integradora y crítica de la acción del ser humano sobre la naturaleza. Entre los conceptos que propone vincular a la germinación este autor, se encuentran: dinámica de poblaciones, tratamientos germinativos de semillas, sitios degradados y no degradados, presencia de depredadores según especie, potencialidad del restablecimiento de plantas nativas. Estas posibilidades de inclusión del contenido "germinación” en una red conceptual más amplia que la específica del proceso de transformación y emergencia de estructuras (tegumento, radícula, 
cotiledones, entre otros) es concordante con la lógica relacional mencionada anteriormente.

Figura 1. Cantidad de publicaciones según la categoría tipo de planta

\section{Origen biogeográfico de la planta}

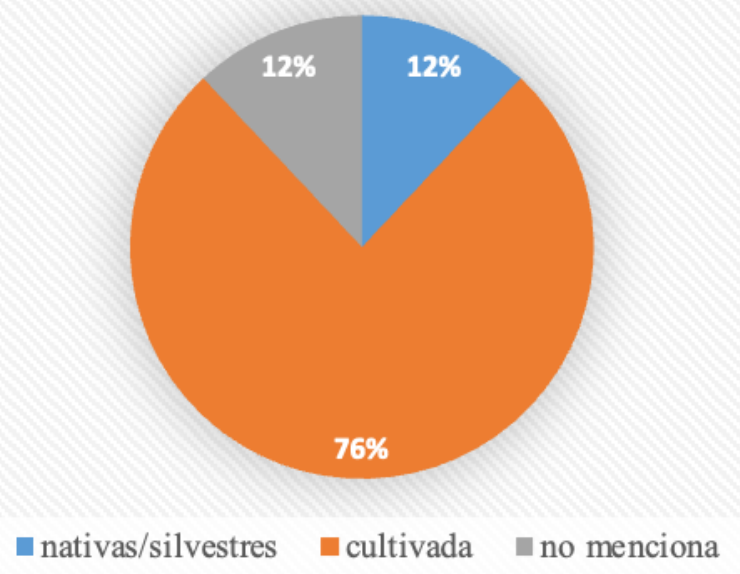

Fuente: Elaboración propia

Los resultados referidos al origen biogeográfico de las especies vegetales utilizadas para enseñar la germinación, pueden explicar la escasa cantidad de trabajos que hacen referencia a mecanismos de dormancia y técnicas para remover ese estado de la semilla (20\%, lo que representa cuatro trabajos). Estos resultados coinciden con los de ALVES (2017), quien, en una revisión de investigaciones publicadas, concluye que, en el nivel superior, no se aborda la enseñanza de la dormición. Aquí resulta fácilmente perceptible, que, si no se trabaja con contenidos sobre adaptación de plantas al ambiente, difícilmente se pueda abordar la enseñanza sobre diferentes mecanismos de dormición para ajustarse a las condiciones de ese ambiente, tema que sistemáticamente se encuentra ausente en las investigaciones como contenido importante en relación a la germinación. Cabe señalar que, entre los trabajos que mencionan la dormición, uno de ellos no especifica la forma de salir de ella y otro no menciona sobre semillas de qué especies de plantas desarrolla la dormición como contenido.

Al analizar la dimensión tema del trabajo (gráfico 2), se observa que la mayoría se focalizan en la enseñanza de habilidades procedimentales (con énfasis en la experimentación con control de variables) y en la anatomía y fisiología de la 
germinación. Respecto al tópico habilidades procedimentales, es de destacar que, diversos autores acuerdan en que la enseñanza de las Ciencias Naturales no implica solamente el cuerpo de modelos científicos, sino también, el metodológico o las destrezas de pensamiento que se desarrollan en las ciencias (ADÚRIZ BRAVO, 2011; CRUJEIRAS PÉREZ; JIMÉNEZ ALEIXANDRE, 2015; VARELA, 2020). Como se evidencia en esta revisión, la germinación es un contenido que básicamente se orienta a la enseñanza de habilidades procedimentales e indagación experimental como manipular variables (luz, agua, aire, entre otras), y observar cambios. Estas actividades son factibles por el hecho de que pueden llevarse a cabo dentro del aula, con poco riesgo para los niños y las niñas, y el tiempo que demanda el proceso de germinación que facilita la observación. Asimismo, resulta adecuado para organizar el cronograma y planificación del profesorado, compartir lecturas complementarias con los estudiantes mientras avanza el desarrollo de estructuras vegetativas, la elaboración de gráficos, entre otras. Las habilidades procedimentales relacionadas con la germinación, se encuentran referidas principalmente al nivel infantil y secundario $(85,7 \%)$, mientras que uno solo de los trabajos (14,3\%), se centran en este tópico en el nivel primario y ninguno en la Formación docente.

Figura 2. Cantidad de publicaciones según categorías de la dimensión tema del trabajo

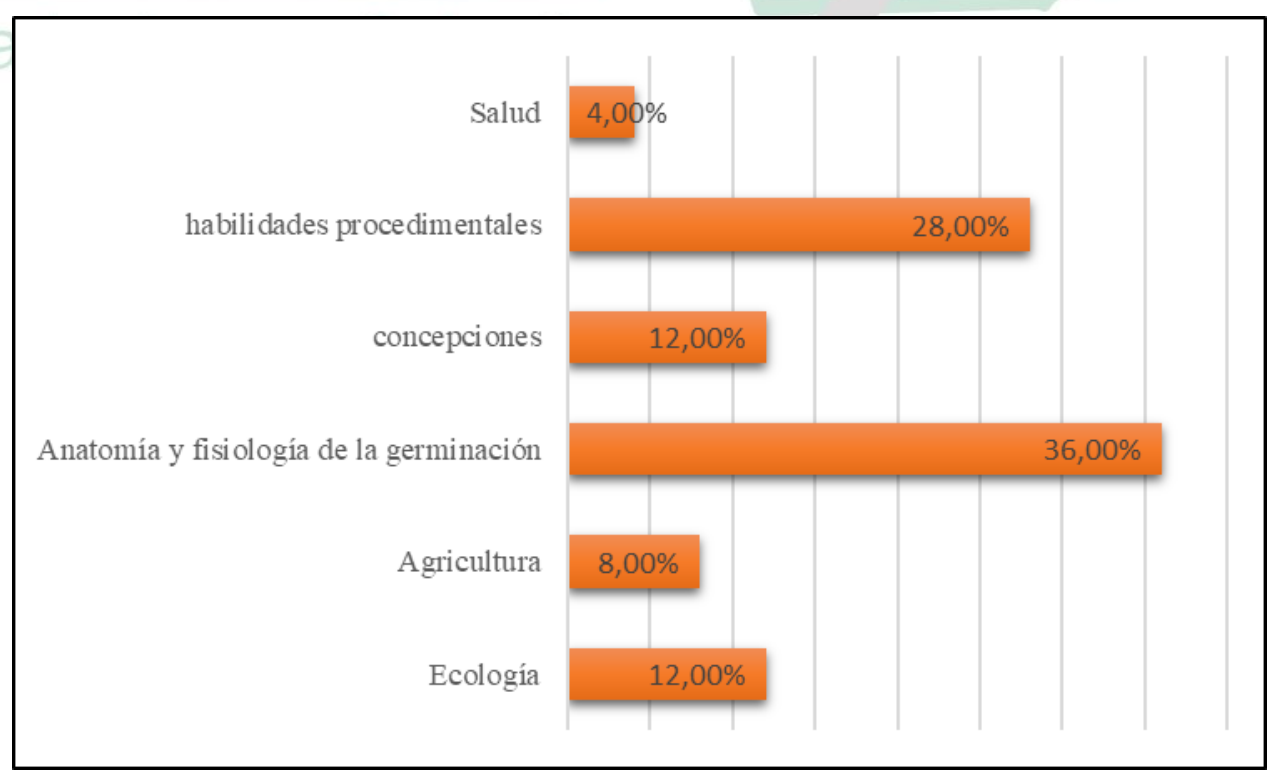

Fuente: Elaboración propia 
Los trabajos en los que el tema central son la fisiología y la anatomía de la germinación, corresponden al nivel primario $(33,4 \%)$, a la formación docente $(44,4 \%)$ y nivel secundario $(22,2 \%)$ y están asociados en todos los casos al germinador de Phaseolus vulgaris. Estos resultados se condicen con los contenidos curriculares de la escuela primaria, pero, al poner la centralidad en los conceptos anatómicos y procesos fisiológicos, sin relacionarlo con el ambiente, puede conllevar el riesgo de adherir implícitamente a una enseñanza tradicional (POZO; CRESPO, 2000) aún en las primeras etapas del sistema educativo. Estos resultados permiten inferir que, mientras que en el nivel inicial los contenidos relacionados con la germinación están asociados a la enseñanza de habilidades procedimentales, en la escuela primaria se centran en la enseñanza de los procesos fisiológicos y anatómicos de la germinación y en el nivel secundario vuelve a ser utilizada como referencia para los procesos científicos.

Respecto a la categoría concepciones, se encontró un trabajo referido a las concepciones de niños y niñas de nivel infantil y primario, uno respecto a estudiantes de nivel secundario y uno referido a las concepciones de docentes que dictan clases en infantil y primaria. Existe abundante bibliografía respecto a las concepciones de niños y niñas sobre plantas, entre las que se encuentran: ideas limitadas de los niños y niñas sobre qué es una planta y como funciona (KINCHIN, 1999), dificultad para reconocerlas como seres vivos (HICKLING; GELMAN, 1995), asociar a las flores con una función estética y no como órgano reproductivo (WARWICK; SPARKSLINFIELD, 1996) y a menudo no considerar a las semillas como parte de una planta hasta que han crecido a partir de ella (ALLEBONE, 1995). El trabajo sobre concepciones infantiles es el de Jewell (numerado como 5 en la tabla 1), y en él, la autora encuentra que los infantes diferencian que es una semilla de aquello que no lo es, pero les cuesta en casos no comunes como el Coco nucifera (coco); y que el 50\% de los más pequeños (de 4 a 8 años) dicen que las semillas no están vivas. La publicación de Lin (numerado como 6), refiere a las concepciones sobre varios tópicos relacionados con las plantas, en estudiantes de nivel secundario y es una referencia importante en la investigación sobre la enseñanza de estos contenidos. Entre los resultados que encuentra, se destaca la escasa comprensión acerca del crecimiento y desarrollo de las plantas, la falta de conocimiento válido sobre la nutrición de las semillas y 
justificaciones tautológicas, teleológicas y antropomórficas respecto a procesos de germinación, fotosíntesis y crecimiento.

Finalmente, el trabajo sobre concepciones docentes es el de Link-Pérez y Schussler (numerado como 15) y trata sobre entrevista para indagar cómo enseñan maestras de nivel infantil y primario, en las cuales se destaca que las docentes comentan que sus propios estudiantes le dicen “¡Basta ya de germinar porotos!” (p.104).

Es notable que sólo dos de los artículos analizados estén relacionados con temas de Ecología, lo que señala un escaso enfoque integrador y de relación con el ambiente. En el marco de este trabajo señalamos como llamativa la ausencia del desarrollo de temáticas de restauración ecológica en las propuestas educativas y que ninguno de los artículos analizados está encuadrado en la educación ambiental. Respecto al tópico Salud, se encontró un artículo (PECASKI MCLENNAN, 2010), que aborda una experiencia didáctica en nivel infantil, la cual trata sobre la siembra en macetas y huertas de semillas de plantas comestibles para incorporarlas luego a la dieta de los infantes.

\section{CONCLUSIONES}

En primer lugar, y como se señaló al principio, en la etapa inicial de una tesis de doctorado, el estudio de antecedentes del tema de investigación exige una búsqueda rigurosa, en la literatura publicada, para detectar los hallazgos en el conocimiento ya construido, como así también, las preguntas aún no resueltas. Al mismo tiempo, permite localizar los grupos de investigación y autores con los cuales se comparten objetivos, metodologías y preocupaciones similares en el campo de la Didáctica de las Ciencias Naturales y generar vínculos e intercambios académicos que alienten y promuevan la formación en posgrado.

En cuanto al estudio de los antecedentes, según consta en documentos curriculares, la germinación ha sido abordada históricamente de manera descriptiva y como proceso generalizado de las plantas fanerógamas (con semillas), sin considerar la diversidad de formas en que las especies germinan según el bioma. Tal es así que muchas semillas "no germinan" debido a la presencia de mecanismos que inhiben la 
germinación conocidos como "dormición o latencia" (BASKIN; BASKIN, 2014). Tampoco ha preponderado una lógica relacional que incluya a las relaciones interespecíficas o el ambiente. Algunos documentos curriculares en la Patagonia Argentina como los de las provincias de Neuquén y Río Negro han comenzado a considerar este enfoque, aunque eso no se refleja en los artículos académicos analizados. Aún en la actualidad, se sigue recurriendo principalmente al poroto (Phaseolus vulgaris), haciendo hincapié en los procesos morfofisiológicos y en las necesidades genéricas de germinación de las semillas sin dormición como tema de análisis y/o investigación desde la lógica disciplinar de la Botánica. Si bien es innegable el buen aporte de la enseñanza de la germinación a la formulación de problemas e indagación, aislar a las semillas del fruto, a los frutos de las plantas, a las plantas de las comunidades bióticas y su ambiente puede resultar en una contradicción con las finalidades de la enseñanza de las Ciencias Naturales y de la Educación ambiental, que enunciamos en apartados de este artículo. Las publicaciones analizadas reflejan la importancia de la enseñanza de habilidades procedimentales en la enseñanza de las Ciencias Naturales. Sin embargo, la mayoría de los diseños experimentales propuestos en los artículos no consideran el ambiente natural de las plantas, con el riesgo de que el trabajo de laboratorio resulte un modelo estereotipado que no responde a problemas reales del contexto socioambiental.

Acordamos con Link-Pérez y Schussler (2013), en que, para una enseñanza efectiva sobre plantas, es necesario proponer actividades con una mayor diversidad de especies y no solo con aquellas cultivables, y ampliar la mirada hacia el ambiente ecológico y cultural del cual niños y niñas forman parte. Esto es particularmente importante en Latinoamérica y el Caribe en donde viven grandes proporciones de campesinos, rica biodiversidad e interés en promover la recuperación de los ambientes degradados (PEREZ; RASSETO; FARINA, 2021). Las comunidades locales que interactúan en la vida real con las plantas de su región, conocen los lugares en donde se encuentran plantas de interés, cuándo se pueden obtener las semillas y cómo se logra que germinen. Hacer participar a las comunidades, familias, padres y contexto socioambiental en el proceso de la germinación se contrapone a los modelos solo centrados en una mirada que podría ser considerada "reduccionista" de la germinación. 
Como se afirmó anteriormente, disciplinas como la restauración ecológica vienen brindando nuevos contextos para enseñar la germinación. En este tema en particular, se ha convocado a los gobiernos de todo el mundo, en el marco de la década de la restauración ecológica 2021-2031, a comprometerse con "una oportunidad única para garantizar que toda una generación de escolares, que serán futuros tomadores de decisiones, entiendan acabadamente los beneficios derivados de los ecosistemas y la necesidad de la restauración ecológica" (UN 2020, p. 8). Desde la educación formal esto se puede lograr, entre otras formas, poniendo atención en semillas de especies nativas de la región, del patio de la escuela o el entorno escolar, haciéndolas germinar en viveros escolares o en el aula, y plantándolas en la misma escuela, en plazas públicas, en hogares de los niños y niñas o en sitios degradados (MCCANN; MILLER, 2004; RASSETTO; FARINA; PEREZ, 2021). Este tipo de propuesta educativa está en la antípoda del repetido germinador y el foco reducido al experimento, que termina habitualmente descartando las plántulas en un recipiente de residuos. Si el interés es enseñar habilidades para desarrollar la experimentación, el contexto de la restauración ecológica y la Educación Ambiental, también ofrece esta oportunidad. Se puede problematizar temas de emergencia y crecimiento de raíces, cotiledones, hojas verdaderas partiendo de la interpretación del ambiente y poner en juego aspectos ecológicos y sociales que se quieran seleccionar y analizar; de la misma forma, estos conocimientos puedan desarrollarse desde la educación basada en la restauración ecológica (MCCANN, 2011). Consideramos que la restauración ecológica puede ser un nuevo "espacio conceptual" para abordar la germinación, la vida de las plantas y el ambiente de una manera integrada. En esta línea de trabajo, la enseñanza de la germinación puede contribuir junto a la restauración a involucrar y beneficiar a las comunidades en la búsqueda de caminos para prevenir la degradación en el futuro y propagar una ética que se extienda y considere a la tierra, como lo propuso Aldo Leopold (2004).

\section{BIBLIOGRAFÍA}

ACHILLI, E. Investigar en antropología social. Los desafíos de transmitir un oficio. Rosario, Argentina: Laborde Editor, 2005. 
ADÚRIZ BRAVO, A. Concepto de modelo científico: una mirada epistemológica de su evolución. En: GALAGOVSKY, L (coord.) Didáctica de la Ciencias Naturales. El caso de los modelos científicos. Buenos Aires, Argentina: Lugar Editorial, 2011.

ALlebOnE, B. Children's Ideas about Plants. Primary Science Review, v. 39, p. 20 23. 1995.

ALVES, A. Germinating sedes. A experiential education in kindergarden. A handbook on experiential Education. Pedagogical Guidelines for teachers and Paters, p. 150155). 2016. Iasi, Rumania: Expeducom. Disponible en: https://bibliotecadigital.ipb.pt/handle/10198/13770. Acceso em: 24 mai. 2021.

ALVES, D. S. Botânica no ensino superior: uma sequência didática para o ensino de dormência e germinação de sementes. 2017. 123. Dissertação (Mestrado Profissional em Ensino) - Universidade Estadual do Norte do Paraná, Cornélio Procópio, 2017.

ARGENTINA. Núcleos de Aprendizajes Prioritarios Nivel Inicial. Ministerio de Educación de la Nación. Buenos Aires, Argentina, 2004.

BASKIN, J.M.; BASKIN, C.C.; LI, X. Taxonomy, anatomy and evolution of physical dormancy in seeds. Plant Species Biology, v. 15, p. 139-152. 2000.

BASKIN, C. Breaking physical dormancy in seeds - focussing on the lens. New Phytologist, v. 158, p. 227-238. 2003.

BASKIN, J.M.; BASKIN, C.C. A classification system for seed dormancy. Seed Science Research, v. 14, p.1-16. 2004.

BASKIN, C.C.; BASKIN, J.M. Seeds: ecology, biogeography, and evolution of dormancy and germination, 2nd edition. California, USA: Academic Press, 2014.

BRAVIN, C. Y PIEVI, N. Documento metodológico orientador para la investigación educativa. Ministerio de Educación de la Nación, Argentina. 2008.

CACHAPUZ, A.F.; LOPES, B.; PAIXÃO, F.: PRAIA, J.F.; GUERRA, C. Reseña del 'Seminario Internacional sobre "El estado actual de la investigación en enseñanza de las ciencias". Revista Eureka sobre Enseñanza y Divulgación de las Ciencias, vol. 3 n. 1, pp. 167-171. 2006. Disponible en:

http://doi.org/10.25267/Rev_Eureka_ensen_divulg_cienc.2006.v3.i1.14. Acceso em: 24 mai. 2021.

CALIXTO FLORES, R. Educación popular ambiental. Trayectorias, v. 12, n. 30. 2010.

Recebido em: 30/05/2021

Aceite em: 13/08/2021 
CRUJEIRAS PÉREZ, B. Y JIMÉNEZ ALEIXANDRE, M.P. Desafíos planteados por las actividades abiertas de indagación en el laboratorio: articulación de conocimientos teóricos y prácticos en las prácticas científicas. Enseñanza de las Ciencias, 33 (1), p. 63-84. 2015. Disponible en: http://doi.org/10.5565/rev/ensciencias.1469._Acceso em: 24 mai. 2021.

CRUZ-GUZMÁN, M, PUIG, M. GARCÍA-CARMONA, A. ¿Qué tipo de actividades diseñan e implementan en el aula futuros docentes de Educación Infantil cuando enseñan ciencia mediante rincones de trabajo?. Enseñanza de las Ciencias, v. 38 n.1, p. 27-45. 2020. Disponible en: https://doi.org/10.5565/rev/ensciencias.2698. Acceso em: 24 mai. 2021.

DUNCAN, C.; SCHULTZ, N.; LEWANDROWSKI, W.; GOOD, M.K.; COOK, S. Lower dormancy with rapid germination is an important strategy for seeds in an arid zone with unpredictable rainfall. PLoS ONE, v. 14n. 9. 2019. Dsiponible em: https://doi.org/10.1371/journal.pone.0218421. Acceso em: 24 mai. 2021.

ESPAÑA. Real Decreto 1603/2006. Ministerio de Educación. 2016. Disponible en: https://www.boe.es/boe/dias/2007/01/04/pdfs/A00474-00482.pdf. Acceso em: 24 mai. 2021.

ESPAÑA. Real Decreto 126/2014. Ministerio de Educación. 2014. Disponible en: https://www.boe.es/buscar/pdf/2014/BOE-A-2014-2222-consolidado.pdf. Acceso em: 24 mai. 2021.

FARINA, J; LOZADA, M.; LADIO, A. Conocimiento y uso de plantas silvestres y cultivadas, comestibles y medicinales, en una población asentada en la espeta árida del noroeste de la Patagonia. (Tesis de Licenciatura)-Centro Regional Universitario Bariloche, Universidad Nacional del Comahue, Bariloche, 2008.

FARINA, J.; ACUÑA, M.I.; PÉREZ, D.R.; RASSETTO, M.J. Marco conceptual y procedimiento para la construcción y validación de un cuestionario sobre las concepciones de enseñanza de las Ciencias Naturales del profesorado de Nivel Inicial. Revista electrónica de Investigación en Educación en Ciencias, v. 14 n.1, 30-38. 2019.

FREIRE, P. La educación como práctica de la libertad. Santiago: ICIRA, 1969.

GARCÍA, J. E. El conocimiento escolar como proceso evolutivo: aplicación al conocimiento de nociones ecológicas. Investigación en la escuela, v. 23, p. 65-76. 1994.

GARCÍA, J. E. Y PORLÁN ARIZA, R. El proyecto IRES (investigación y Renovación Escolar). Revista Bibliográfica de Geografía y Ciencias Sociales, v. 205, p. 1-16. 2000. 
GARZÓN, N.V.; RODRÍGUEZ LEÓN, C. H.; CECCON, E.; PÉREZ, D.R. Ecological restoration-based education in the Colombian Amazon: Toward a new society-nature relationship. Restoration ecology, June 2020. Disponible en: https://doi:10.1111/rec.13216. Acceso en: 20 mai. 2021.

HAMMANN, M.; HOL PHAN, T.; EHMER, M.; GRIMM, T. Assesing pupils' skills in experimentation. Journal of Biological Education, v. 42 n. 2, p. 66-72. 2008.

Disponible en: https://doi.org/10.1080/00219266.2008.9656113. Acceso en: 20 mai. 2021.

HICKLING, A. K.; GELMAN. S.A. How Does Your Garden Grow? Early Conceptualization of Seeds and Their Place in the Plant Growth Cycle. Child Development, v. 66, p. 856-876. 1995.

HILLISON, J. Agriculture in the classroom: Early 1900s Style. Journal of Agricultural Education, v. 39 n. 2, p. 11-18. 1998.

JEWELL, N. Examining Childen's Models of Seed. Journal of Biological Education, 36 (3), p. 116-122. 2002.

KAUFMAN, V.; SERULNICOFF, A. Conocer el ambiente. Una propuesta para las Ciencias Sociales y Naturales en el Nivel Inicial. En: MALAJOVICH, A. (Comp.)

Recorridos didácticos en la educación inicial. Buenos Aires, Argentina: Paidós, 2000.

KINCHIN, I. M. Investigating Secondary-School Girls' Preferences for Animals or Plants: A Simple 'Head-To-Head'. Comparison Using Two Unfamiliar Organisms. Journal of Biological Education, v. 33, p. 95-99. 1999.

LEFF, E. racionalidad ambiental y diálogo de saberes. Significancia y sentido en la construcción de un futuro sustentable. Revista Polis, v. 7, p. 1-35, 2004. Disponible en: https://journals.openedition.org/polis/6232. Acceso en: 16 jun. 2021.

LEOPOLD, A. C. Living with the Land Ethic. BioScience v. 54 n. 2, p. 149-154. 2004.

LINK-PÉREZ, M.; SCHUSSLER, E. Elementary botany: how teachers in one school district teach about plant. Plant Science Bulletine, v. 59 n. 3, p. 99-110. 2013. Disponible en: https://doi 10.3732/psb.1300002._Acceso en: 20 mai. 2021.

MARQUES DE ABREU, A.H.; DA SILVA ABEL, E.L.; BENEVIDES BITTENCOURT, C.S.; GAMA ALVES, A.; DUARTE FERREIRA, A. Replantando vida: la restauración forestal como herramienta para la rehabilitación humana. In:

Beyond restoration ecology: social perspectives in Latin America and the Caribbean. Buenos Aires, Argentina: Vázquez Mazzini Eds, p. 89-100. 2017.

MCCANN, E. Restoration-Based Education: Teach the Children Well. In: Egan, D.; Hjerpe, E. E.; Abrams, J. (Eds). Human dimensions of ecological restoration. Washington, U.S.: Island Press, p. 315-335. 2011

Recebido em: 30/05/2021

Aceite em: 13/08/2021 
MCCANN, L.; MILLER, T. Ecological Restoraation-based Education Transforms Schoolgrounds and Education. Proceedings of the North American Prairie Conferences, 19, p. 200-204. 2004. Disponible en : https://digitalcommons.unl.edu/napcproceedings/87. Acceso en: 20 mai. 2021.

MCCANN E. Restoration based education: teach the children well. En: EGAN D; HJERPE EE; ABRAMS J. (Eds.) Human dimensions of ecological restoration. Washington, U.S.: Island Press. p. 315-335, 2011.

NEWMAN, A. Inclusive Urban Ecological Restoration in Toronto, Canada Allegra. In: Human dimensions of ecological restoration. Washington, U.S.: Island Press, p. 6377, 2011.

NOVO, M. La educación ambiental, una genuina educación para el desarrollo sostenible. Revista de Educación, número extraordinario, p. 195-217. 2009.

PECASKI MCLENNAN, D. "Ready, set, grow!" Nurturing Young children throught gardening. Early Childhood Educ J, v. 37, p. 329-333. 2010. Disponible en: http://doi10.1007/s10643-009-0366-4. Acceso en: 20 mai. 2021.

PÉREZ, D.; GONZÁLEZ, F.; RODRIGUEZ ARAUJO, M.; PAREDES, D.; MINARDI, E. Restoration of Society-Nature Relationship Based on Education: A Model and Progress in Patagonian Drylands. Ecological Restoration, v. 37 n. 3, p. $182-$ 191. 2019.

PORLÁN ARIZA, R. Hacia un modelo de enseñanza - aprendizaje de las ciencias por investigación. En KAUFMAN, M.; FUMAGALLI, L. (Comp.) Enseñar Ciencias Naturales. Reflexiones y propuestas didácticas. Buenos Aires, Argentina: Paidós Educador, 1999.

POZO, J. I.; CRESPO, M.A. Aprender y enseñar ciencias. Madrid: Morata, 2000.

POZZOLI, M.T. Transformar el conocimiento en la sociedad globalizada. Pensamiento complejo y transdisciplinariedad. Polis Revista Latinoamericana, v. 16, p. 1-26. 2007.

RÍO NEGRO (ARG.) Diseño Curricular para la Educación Inicial. Res. 2121/19 Ministerio de Educación y Derechos Humanos de Río Negro, Viedma, Argentina. 2019.

RÍO NEGRO (ARG.) Diseño Curricular Nivel Primario. Res. 2135/11. Consejo Provincial de Educación. Río Negro. Argentina. 2011.

RODRIGUEZ ARAUJO, M.E., PÉREZ, D.R., BONVISSUTO, G.L. Seed germination of five Prosopis shrub species (Fabaceae-Mimosoideae) from the Monte and Patagonia phytogeographic provinces of Argentina. Journal Arid Environ, v. 147, p. 159-162. 2017.

Recebido em: 30/05/2021

Aceite em: 13/08/2021 
ROMERA, M. J. La investigación-acción en didáctica de las ciencias: perspectiva desde las revistas españolas de educación. Enseñanza de las Ciencias, v. 32 n.1, p. 221-239. 2014. Disponible en: http://dx.doi.org/10.5565/rev/ensciencias.529. Acceso en: 20 mai. 2021.

STATU, R.; BONIOLO, P.; DALLE, P.; ELBER, R. Manual de Metodología: Construcción del Marco Teórico, formulación de los objetivos y elección de la metodología. Buenos Aires, Argentina: CLACSO Libros Editores, 2005

SUAREZ SILVA, J.A.; FORTES BRAIBANTE, M.E. Aprendizagem significativa: concepções na formação inicial de professores de Ciências. Revista Insignare Scientia, v. 1 n.1, p. 1-22. 2018.

TRÉLLEZ, E. Algunos elementos del proceso de construcción de la Educación Ambiental en América Latina. Revista Iberoamericana de Educación, v. 41, p. 68-81. 2006.

UN. United Nations. Strategy of the United Nations Decade on Ecosystem

Restoration. 2020. Disponible en: https://decaderestoration.org. Acceso en: 21 mai. 2021.

VARELA, P. Aprender ciências por investigação na educação pré-escolar: exploração de uma proposta didática em contexto de formação inicial de educadores. Revista Insignare Scientia, v. 3 n.1, p. 357-375. 2020.

VIDAL, M.; MEMBIELA, P. On teaching the scientific complexity of germination: a study with prospective elementary teachers. Journal of Biological Education, v. 48 n.1, p. 34-39. 2014.

WARWICK, P.; SPARKS-LINFIELD, R. Speeding Up Plant Growth and Children's Ideas. Primary Science Review, v. 43, p. 26-29. 1996. 